\title{
Comparison of Numerical Methods for SWW Equations
}

\author{
Leonard Bezati $^{1}$, Shkelqim Hajrulla ${ }^{2}$, Besiana Hamzallari ${ }^{3}$ \\ ${ }^{1,2}$ University of Vlora, Albania \\ ${ }^{1,3}$ University of Tirana, Albania
}

\begin{abstract}
:
In this paper we consider three methods of approximation for the nonlinear water wave equation. In particular we are interested of $\mathrm{KdV}$ equation as a stationary water wave. The first is the method of approximation with a polynomial, the second method is the finite-volume method and the third method is Laplace decomposition method (LDM). A comparison between the methods is mentioned in this article.

We treat the considered methods comparing the obtained solutions with the exact ones. We give in particular the numerical results compared with the analytical results. We show that the used methods are effective and convenient for solving the water wave equations. We can propose and sure that the method of approximation with a polynomial gives accurate results.
\end{abstract}

Keywords: KdV-Bezuci equation, approximation, finite-volume method, the method of lines, LDM method.

\section{Introduction}

We consider the initial-boundary value problem associated with the nonlinear dispersive and dissipative wave which was formulated by Korteweg, de Vries and Burgers in the form (see [2])

$$
\frac{\partial U}{\partial x}+\mu u \frac{\partial U}{\partial x}-\theta \frac{\partial^{2} U}{\partial x^{2}}+\delta \frac{\partial^{3} U}{\partial x^{3}}=0
$$

where $\mu, \theta, \delta$ are constant coefficients.

In [1] we see the change form of long waves in a rectangular zone and on a new type of stationary waves. In particular, we deal with the nonlinear wave equation, named Korteweg de Vries-Bezuci form as follow:

$$
\frac{\partial U}{\partial x}-\delta \frac{\partial u}{\partial x}+\mu u \frac{\partial u}{\partial x}-\theta \frac{\partial^{2} u}{\partial x^{2}}=0
$$

where, the coefficients $\mu$ and $\theta$ in Eq. (1.3) represent the damping and the dispersion coefficients, respectively and $\delta=\frac{p_{z}(x, y)}{S}$ is the wave breaking coefficient.

Numerical methods, numerical results and comparisons are considered in this article. It is well known that many physical phenomena can be described by the Korteweg-de Vries-Bezuci equation. Eq. (1.2) can serve as a nonlinear wave model of a fluid in an elastic tube [1] and turbulence [3,4].

Johnson [5], Demiray [6] and Antar and Demiray [7] derived KdVB equation as the governing evolution equation for waves propagating in fluid-filled elastic or visco-elastic tubes in which the effects of dispersion, dissipation and nonlinearity are present.

Numerical methods for solutions of the KdV equation are known since long time. Recall here [8, 9, 10]. Many problems, however, involve not only dispersion but also dissipation, and these are not governed by the $\mathrm{KdV}$ equation. Other cases regarded the governing evolution equation can be shown to be the so-called Korteweg-de Vries-Bezuci equation. 


\section{Fundamentals of Modified Laplace Decomposition Method}

In this section, a brief outline of LDM is explained [10]. For this, we consider the general nonlinear partial differential equation of first order (without loss of generality) in the following form:

$$
L u(x, t)+R u(x, t)+N(u(x, t))=h(x, t)
$$

with the following initial condition:

$$
u(x, 0)=f(x)
$$

where $L$ is the first-order differential operator, $L=\partial / \partial t, N(u)$ presents the nonlinear term, $h(x, t)$ is the source term and. $R$ is the remainder of the linear operator. Thus we get

$$
L(u)=g(t)-R(u)-N(u)
$$

The methodology consists of applying Laplace transform first on both sides of

$$
L[L u(x, t)]+L[R u(x, t)]+L[N(u(x, t))]=L[h(x, t)]
$$

We discuss the solution of the KdV-Bezuci equation using LDM. Eq. (1.2) can be written in an operator form:

$$
L u(x, t)=\delta u_{x x}-\mu u u_{x}-\theta u_{x x}
$$

where the differential operator $L$ is $L=\partial / \partial t$. We will define the solution $\mathrm{u}(x, \mathrm{t})$ by the series in the form:

$$
u(x, t)=\sum_{n=0}^{\infty} u_{n}(x, t)
$$

and the nonlinear operator $N(u)$ represented by an infinite series of the so-called Adomian's polynomials:

$$
N(u)=\sum_{n=0}^{\infty} P_{n}(x)
$$

where $u_{n}(x, t), n \geq 0$ are the components of $u(x, t)$ that will be elegantly determined and $P_{n}$ are called Legendre's polynomials, which are a system of complete orthogonal system with respect to the weight function $v(x)=1$ over the interval $-1 \leq x \leq 1$. That is a polynomial of degree $\mathrm{n}$, such that

and defined by Rodrigues's formula

$$
\int_{-1}^{1} \mathrm{P}_{\mathrm{m}}(\mathrm{x}) \mathrm{P}_{\mathrm{n}}(\mathrm{x}) d x=0 \text { if } n \neq m
$$

$$
P_{n}=\frac{1}{2^{n} n !}\left[\frac{d^{n}}{d \lambda^{n}}\left(\sum_{i=0}^{\infty}\left(\begin{array}{l}
n \\
k
\end{array}\right)^{2} \lambda^{i} u_{i}\right)\right]_{\lambda=0}, \quad n \geq 0
$$

that represent the nonlinear term $u u_{x}$ and given by

$$
\begin{aligned}
& P_{0}=u_{0 x} u_{0} \\
& P_{1}=u_{0 x} u_{1}+u_{1 x} u_{0} \\
& P_{2}=u_{0 x} u_{2}+u_{1 x} u_{1}+u_{2 x} u_{0} \\
& P_{3}=u_{0 x} u_{3}+u_{1 x} u_{2}+u_{2 x}
\end{aligned}
$$


The inverse operator $\mathrm{L}^{-1}$ is an integral operator defined by

$$
\begin{gathered}
L^{-1}=\int_{0}^{t}(.) d t \\
U=f_{0}+L^{-1}(g(t)-R(u)-N(u))
\end{gathered}
$$

where $f_{0}$ Is the solution of homogenous equation $L(u)=0$

The first few components of $u_{n}(x, t)$ follows as

$$
\begin{aligned}
& u_{0}(x, t)=f(x) \\
& u_{1}(x, t)=L^{-1}\left(-P_{0}+\theta u_{0 x}-\delta u_{0 x x}\right) \\
& u_{2}(x, t)=L^{-1}\left(-P_{1}+\theta u_{1 x}-\delta u_{1 x x}\right) \\
& u_{3}(x, t)=L^{-1}\left(-P_{2}+\theta u_{2 x}-\delta u_{2 x x}\right)
\end{aligned}
$$

The scheme in (2.12) can easily determine the components $u_{n}(x, t), n \geq 0$. So it is possible to calculate more components in the decomposition series to enhance the approximation. Using initial condition and plotting the solution of KdV-Bezuci equation at $\mathrm{t}=0.01,1,2,2.5$ by using the fundamentals of modified Laplace decomposition method we get,
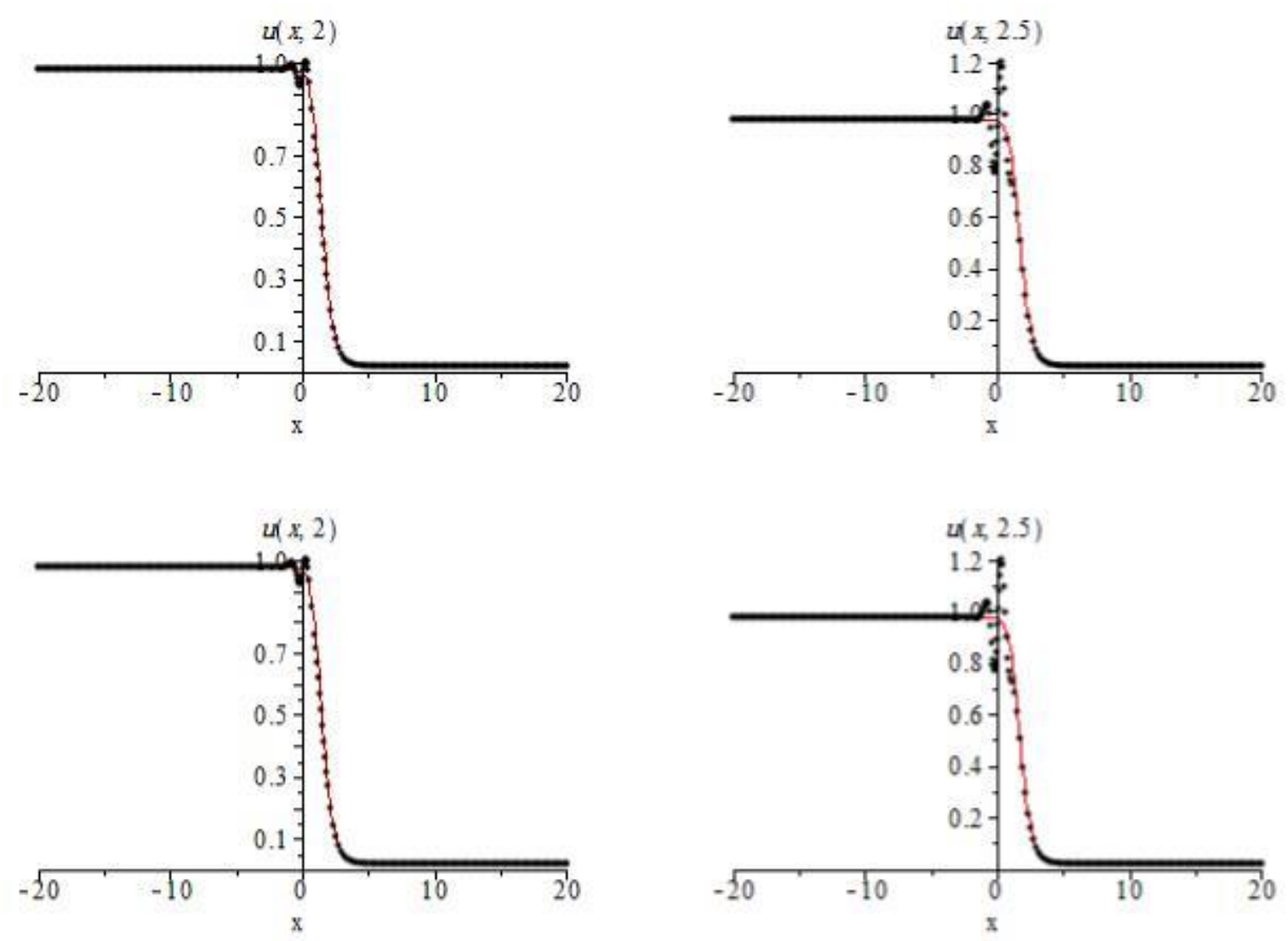

Fig. 1 Comparison of LDM (dotted line) and exact (solid line) solutions corresponding to KdV-Bezuci equation at $\mathrm{t}=0.01,1,2$ and 2.5 where $-20 \leq x \leq 20$. 

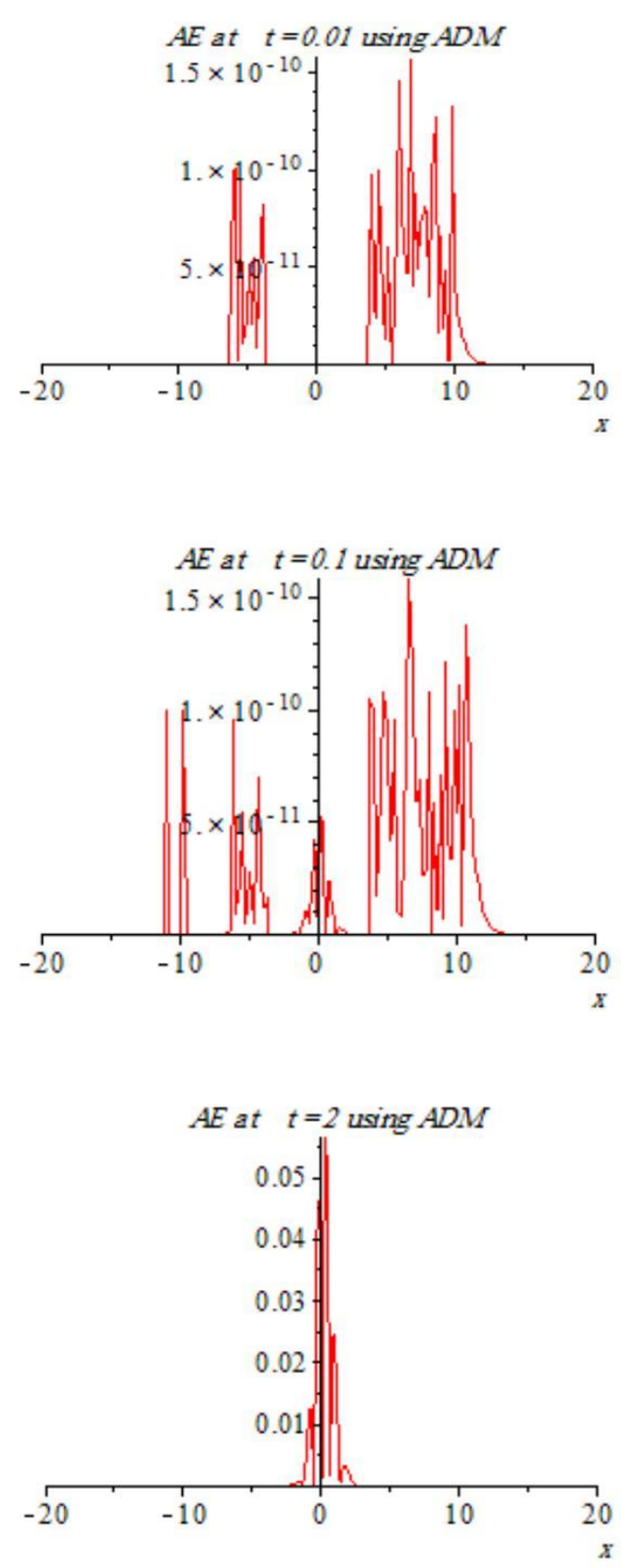
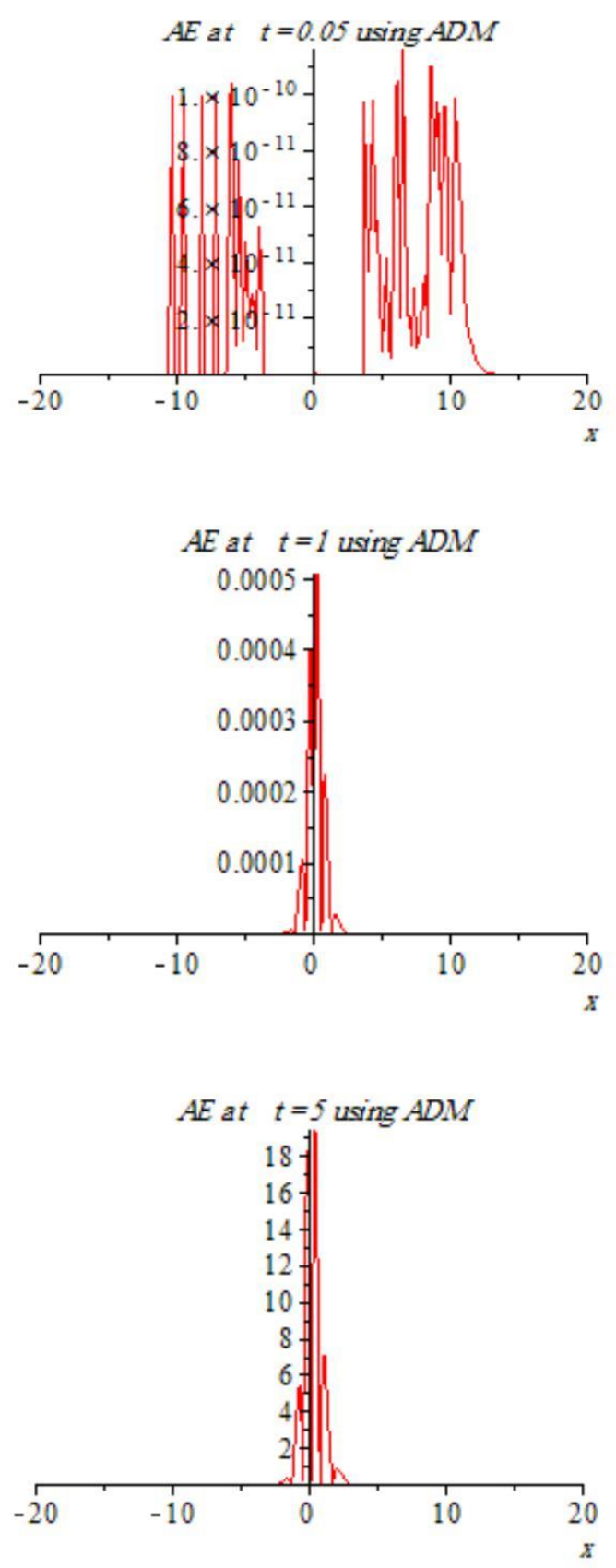

Fig. 2 The absolute error between the exact solution $u(x, t)$ and the (LDM) solution using seven terms for KdV-Bezuci equation at $t=0.01,0.05,0.1,1,2$ and 5 where $-20 \leq x \leq 20$.

\section{The Method of Lines}

The MOL (The method of lines) has the merits of both the finite difference method and analytical method, it does not yield spurious modes nor have the problem of relative convergence. This method [11] is a well established numerical technique for the analysis of transmission lines, waveguide [12-13]. This method usually proceeds in two separate steps: first, approximating the spatial derivatives. Second, the resulting system of semi discrete (discrete in space-continuous in time) ordinary differential equations (ODEs) is integrated in time. The essence of the method of lines is a way of approximating PDEs by ODEs. Obviously, an advantage of the MOL is that one can use all kinds of ODE solvers and techniques to solve the semidiscrete ODEs directly.

The method of lines is regarded as a special finite difference method but more effective with respect to accuracy and computational time than the regular finite difference method. The MOL is generally recognized as a comprehensive and powerful approach to the numerical solution of time-dependent partial differential equations (PDEs). 


\subsection{Solving the KdV-Bezuci equation using the Method of Line}

Let' $\mathrm{s}$ we study our problem in the rectangle zone $a \leq x \leq \leq b, 0 \leq t \leq T$.

Consider KdV-Bezuci equation (1.2) with the initial condition

$$
u(x, 0)=\left(\frac{25 c}{\mu}-100 \mu^{2} \delta+\frac{\theta^{2}}{\delta}\right)+12 \mu^{2} \delta \sin ^{2}(\mu x)-\frac{12}{5} \mu \theta \tan (x)
$$

and the boundary conditions

$$
u(a, t)=0,98, u(x, 0)=0,02
$$

The exact solution is given by

$$
u(x, t)=\left(\frac{25 c}{\mu}-100 \mu^{2} \delta+\frac{\theta^{2}}{\delta}\right)+12 \mu^{2} \delta \sin ^{2}(\mu x-c t)-\frac{12}{5} \mu \theta \tan (\mu x-c t)
$$

The solution domain is the rectangle $a \leq x \leq \leq b, 0 \leq t \leq T$.

The solution using a second order finite difference scheme for $u_{x}$ and $u_{x x}$ is denoted by Method Of Line II.

The derivative $u_{x}$ in KdV-Bezuci equation (1.2) is computed by finite differences scheme in second order approximations $u_{x}=\frac{u_{i+1}-u_{i-1}}{2 h}+O\left(h^{2}\right)$.

The derivative $u_{x x}$ in $\mathrm{KdV}$-Bezuci equation (1) is computed by finite difference scheme in second order approximations $u_{x x}=\frac{u_{i-1}-2 u_{i}+u_{i+1}}{h^{2}}+O\left(h^{2}\right)$.

Applying the above finite difference schemes to Eq. (1.2) yields a system of ordinary differential equations for the unknown $u_{i}$ as functions in $t$ as follows:

$$
\frac{d u_{i}(t)}{d t}=f\left(u_{i}\right), i=1, \ldots \ldots, N-1
$$

Thus, we have the system of differential equations of one independent variable t. This system can be easily solved by using fourth order Runge-Kutta scheme.

$$
\begin{gathered}
U^{n+1}=U^{n}+\frac{\Delta t\left(K_{1}+K_{2}+2 K_{3}+K_{4}\right)}{6} \\
K_{1}=F\left(U^{n}\right), \quad K_{2}=F\left(U^{n}+\frac{\Delta t}{2}\right) K_{1}, \quad K_{3}=F\left(U^{n}+\frac{\Delta t}{2}\right) K_{2}, \quad K_{4}=F\left(U^{n}+\Delta t K_{3}\right)
\end{gathered}
$$

The results obtained using the method of lines have been compared with the exact solution as a plots of the solution and the absolute error (AE) profiles of the $\mathrm{KdV}$-Bezuci equation where $\theta$ and $\delta$ are constants at $c=0.5, \Delta t=10^{-3}, v=\theta / 10 \delta, t \in[0,30]$.

We obtain the Method of line solutions of $\mathrm{KdV}-$ Bezuci equation with high accuracy. The obtained results demonstrate the reliability of the MOL and its wider applicability to nonlinear evolution equations. 

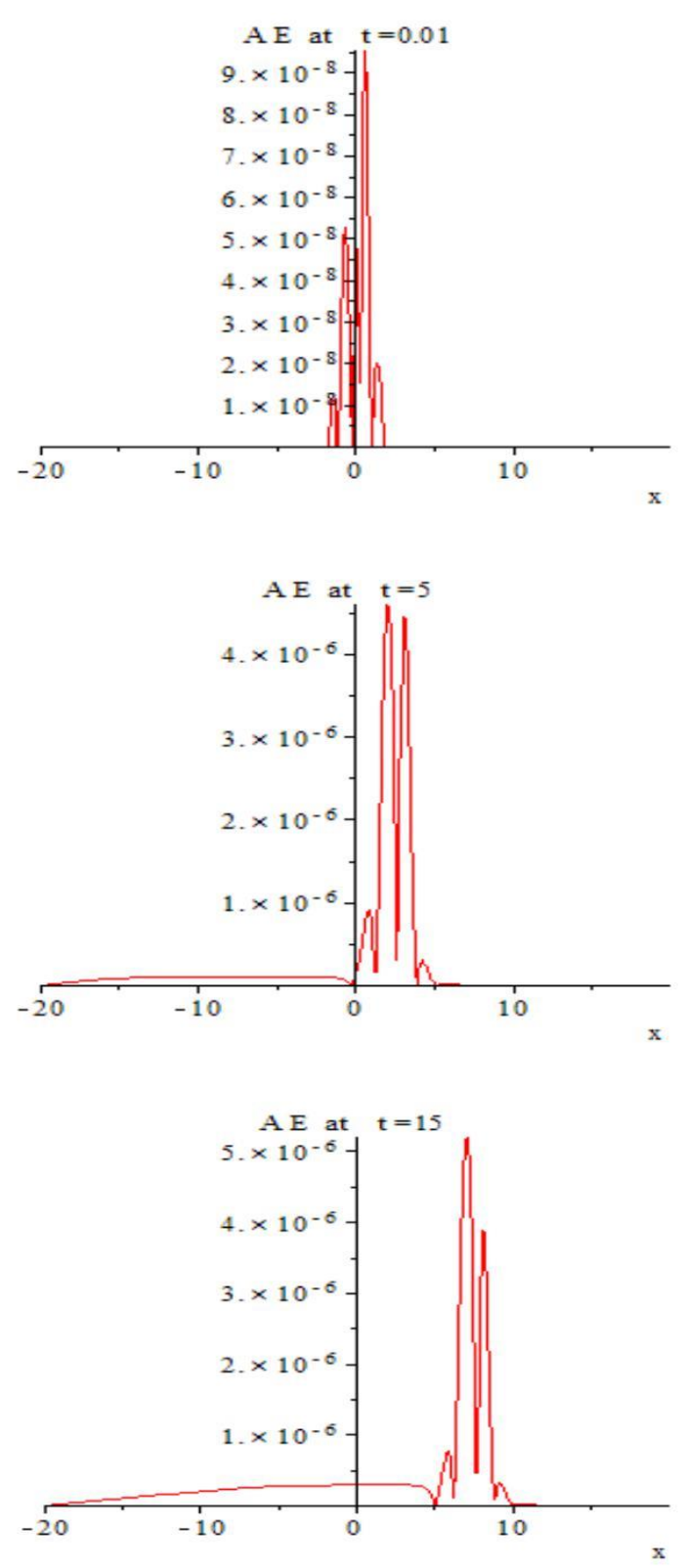
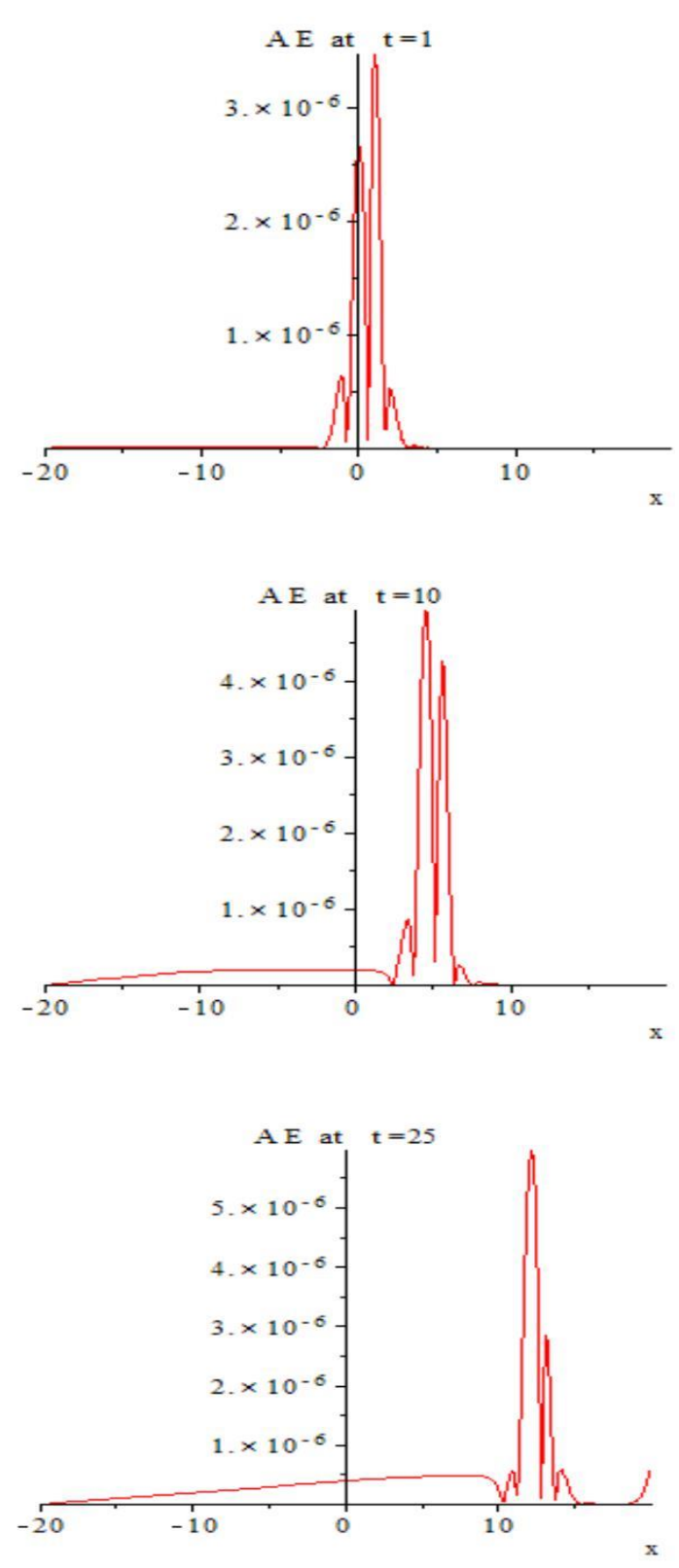

Fig. 3: Comparison of Method of line(dotted line) and exact (solid line) solutions at $N=500, \delta=0.02, \theta=$ $0.2, c=0.5, v=\theta / 10 \delta, \tau \in[0,30]$. 

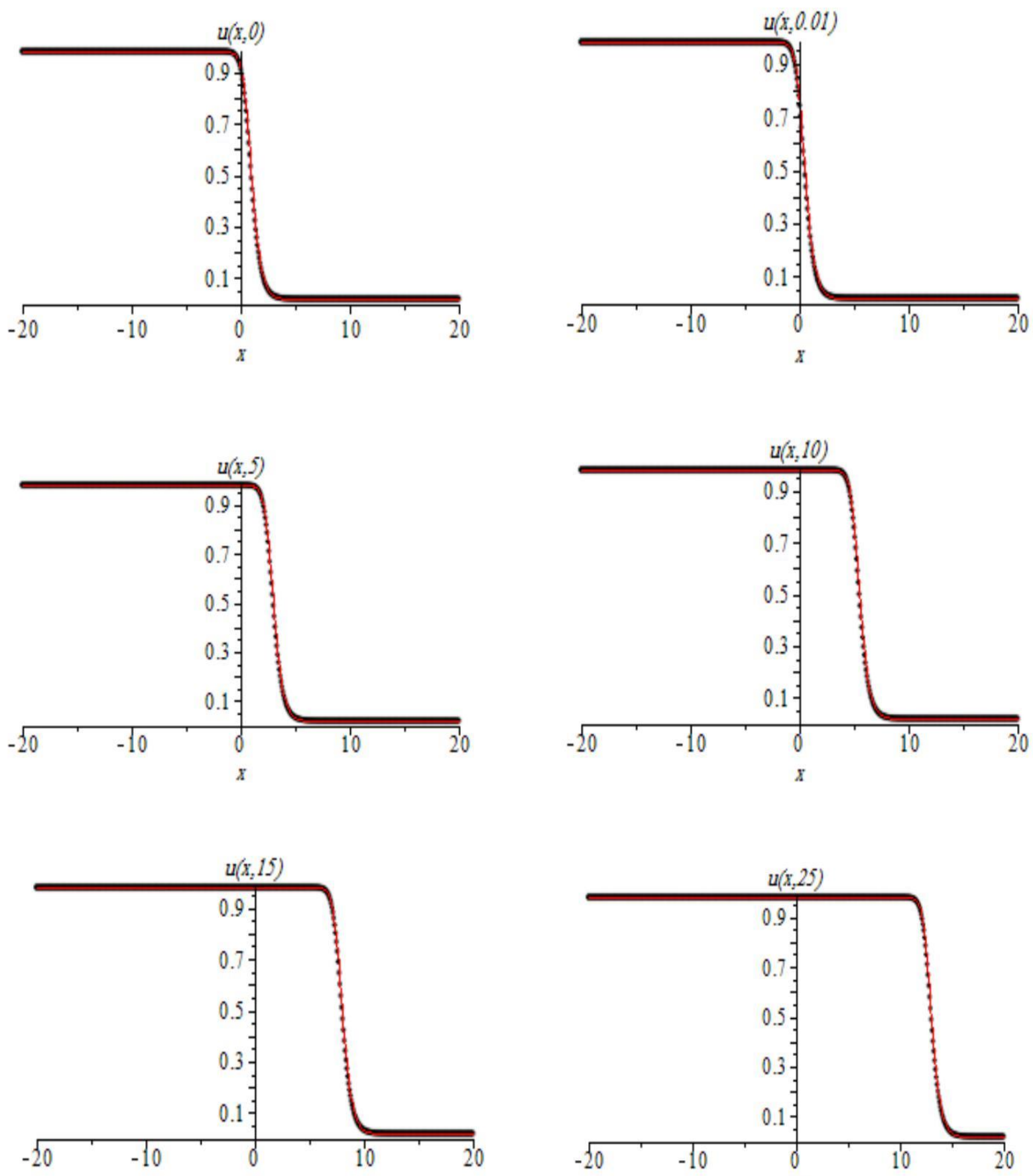

Fig. 4: The absolute error betwểen the exact solution $u(x, t)$ and the (MOL I) solǘtion for KdV-Bezuci equation for $t \in[0,30]$.

\section{Conclusion}

In this article, we conclude that the nonlinear $\mathrm{KdV}-$ Bezuci equation gives solution, which represents an important application in physical problems. The computations associated here were performed using Maple. We have presented the following tables to describe the absolute errors between the exact and numerical solutions. The tables illustrate the errors for both methods, the Laplace decomposition method and the method of lines compared with the exact solution, at different values of $t$.

A review of the decomposition method [14] and a new algorithm for calculating Legendre polynomial for nonlinear operators [15] we have presented. The method of lines and Laplace decomposition method have been implemented for obtaining solutions of the KdV-Bezuci equations. The results show that the considered methods are powerful mathematical tools for obtaining accurate solutions. A comparison between MOL and LDM shows that the accuracy of the MOL is better than that in the LDM for solutions when the time increase.

A comparison between the numerical MOL and the decomposition methods with those obtained by exact solution are given for $\Delta t=10^{-3}$. From the tables, we can observe that the decomposition method is accurate 
as compared with MOL at small period of time but with increasing the time, the MOL is more accurate when compared with LDM. It is observed that if we increase the number of terms in algorithm, the size of calculation is maximized with no increase in accuracy so the reduction of terms facilities the construction of Legendre polynomials for nonlinear operators and gives the same accuracy. LDM can provide the solution with minimal number of iterations.

From the comparative study between LDM and the MOL we may conclude that the MOL is more accurate than LDM.

From the above tables we can infer that LDM have better convergence at small $t$. However, a closer look at the errors of LDM reveals that the error considerably increments with increasing the time. This is an indication of little stability on the part of LDM, in contrast to the MOL.

\section{References}

[1] D. J. Korteweg and G. de Vries, "On the change form of long waves in a rectangular canal, and on a new type of long stationary waves," Philosophical Magazine, vol. 39, p. 422, 1895. View at Google Scholar.

[2] R.S. Johnson, "Nonlinear waves in fluid-filled elastic tu bes and related problems", PhD thesis, University of London, London, (1969).

[3] D.J. Korteweg, de Vries," On the Change of Form of Long Wav es Advancing in a Rectangular Canal and on a New Type of Long Stationary Waves", Philosophical Magazine, 39,422-443 (1 895).

[4] M.J. Ablowitz and P.A. Carkson, Solitons," Nonlinear Evolution Equations and Inverse Scatting”, Cambridge Univer sity Press, 200 (1991).

[5] A.R.Johnson, "nonlinear equation incorporating damp ing and dispersion", Journal of Fluid Mechanics,42,49-60 ( 1970).

[6] H. Demiray," Nonlinear waves in a thick walled viscoelastic tube filled with an inviscid fluid", International Journ al of Engineering Science, 36,359-62 (1998).

[7] N. Antar, H. Demiray, "Nonlinear waves in an inviscid fluid contained in a pre-stressed thin viscoelastic tube", Jou rnal of Applied Mathematics and Physics, 48 ,325-340 (1997).

[8] Bezati, L., Hajrulla, S., \& Hoxha, F. (2018). Finite Volume Methods for Non-Linear Equations. International Journal of Scientific Research and Management, 6(02), M-2018.

[9] S.K. Liu," New periodic solutions to a kind of nonlinear w ave equations", Acta Physica Sinica , 51, 10-14 (2002).

[10] Mehdi Dehghan," Application of the Adomian decomposition method for two-dimensional parabolic equation subject to nonstandard boundary specifications", Applied Mathematic s and Computation, $157,549-560$ (2004).

[11] W.E. Schiesser, "The numerical method of lines: integration of partial differential equations", San Diego, California: Academic Press; (1991).

[12] W. D. Yang and R. Pregla, "Method of lines for analysis of waveguide structures with multi discontinuities”, Electronics Letters ,31 , 892-893 (1995).

[13] R. Pregla and E. Ahlers, "Method of lines for analysis of discontinuities in optical waveguides", Electronics Lett ers, October, $29,1845-1851$ (1993).

[14] G. Adomian, "A review of the decomposition method in applied mathematics", Journal of Mathematical Analysis and Applications, 135 , 501-544 (1998).

[15] A.M. Wazwaz, "A new algorithm for calculating Adomian polynomial for nonlinear operators", Applied Mathematics a nd Computation ,17 (111), 53-69 (2000). 